\title{
ORIGINAL ARTICLE \\ Reliability of infrared thermography in skin temperature evaluation of wheelchair users
}

\author{
I Rossignoli $^{1,2,3}$, PJ Benito ${ }^{2}$ and AJ Herrero ${ }^{3}$
}

Study design: Test-retest reliability analysis segmented by body parts.

Objectives: To examine the reliability of infrared thermography (IRT) in wheelchair users (WCUs), as a noninvasive and risk-free technique to detect the natural thermal radiation emitted by human skin and to allow subsequent interpretations of temperature distributions.

Setting: Research Center on Physical Disability of Valladolid, ASPAYM Castilla y León Foundation, Spain.

Methods: Twenty-four manual WCUs (22 men, 2 women) participated in this study. Skin temperature (Tsk) of 16 and 20 areas of the front and rear upper body, respectively, were measured using an infrared camera. Two thermographic images were recorded in each session during two days separated by $24 \mathrm{~h}$. Results were analyzed using descriptive statistics, intraclass correlation coefficients, coefficients of variation and side-to-side skin temperature differences ( $\delta T s k)$.

Results: The intraclass correlation coefficient produced values ranging from 15 to 95 for the various regions of interest. The coefficient of variation ranged between $1.05-6.18 \%$. The mean Tsk of the front upper body was $31.92^{\circ} \mathrm{C}$, and that of the rear upper body was $31.77^{\circ} \mathrm{C}$. The total $\delta$ Tsk was $0.09 \pm 0.44^{\circ} \mathrm{C}, r=0.583$.

Conclusion: The reliability of IRT varies depending on the analyzed areas. IRT is a noninvasive and noncontact technique that allows measuring the temperature of the skin, with which to advance in WCU research.

Spinal Cord (2015) 53, 243-248; doi:10.1038/sc.2014.212; published online 25 November 2014

\section{INTRODUCTION}

Individuals who use wheelchairs as their main means of mobility have a high incidence of shoulder pain owing to overuse and repetitive propulsion movement. The most commonly used assessment tools for shoulder pain in wheelchair users (WCUs) are questionnaires (mainly the Wheelchair Users Shoulder Pain Index (WUSPI) and Shoulder Pain and Disability Index (SPADI)), physical examination, inclinometer or dynamometer, electromyography and ultrasound. In addition, there are various diagnostic imaging methods widely used for the detection of shoulder pathologies (i.e. radiography, arthroscopy, computed tomography and magnetic resonance imaging). Thurston et al. ${ }^{1}$ argued the need for a noninvasive but accurate test to appropriately assess shoulder pain, suggesting thermography as a well-established technique for joint pain evaluation.

Infrared thermography (IRT) provides information about physiological processes by examining, recording and interpreting skin temperature (Tsk) distributions. ${ }^{2}$ There is a high correlation between both sides of the body; therefore thermal asymmetry in body areas indicates dysfunction, whereas increased heat is mainly associated with inflammation or infection. ${ }^{3}$ IRT has been applied successfully in the field of industry, construction and in a wide range of clinical applications, ${ }^{3}$ like the diagnosis of sports injuries. ${ }^{4}$ IRT is commonly used to estimate surface body temperature, and its reliability has been studied in healthy subjects. ${ }^{5-8}$ Burnham et al. ${ }^{8}$ compared the reliability of three thermometers, finding that the infrared skin device was the most responsive (intraclass correlation coefficient (ICC) 0.97) for the hand, forearm, shoulder, thigh, shin and foot. The intra-examiner reproducibility of a study by Zaproudina et al. ${ }^{5}$ varied from poor in the fingertips to moderately high in the core areas, with a mean ICC of 0.47 . However, no study has analyzed the reliability of IRT in the upper body of WCUs. The special characteristics of people with disabilities, such as sweating and thermoregulation problems, blood distribution or drug intake, make it necessary to study the factors affecting the application of IRT in WCUs.

Thermography requires standardized protocols and caution with the interpretation of thermograms, to avoid confusing past problems with current ones. ${ }^{2}$ Environmental factors (such as ambient temperature, humidity, atmospheric pressure or source radiation), individual factors (both intrinsic: such as gender, age, anthropometry, or skin emissivity, and extrinsic: such as intake factors, therapies or physical activity) and technical factors (such as protocol or camera features) ${ }^{9}$ have to be considered. In order to know if the technique is reproducible in standard conditions, it is necessary to analyze the reliability. Therefore, the aim of this article is to examine the reliability of IRT in WCUs.

\section{MATERIALS AND METHODS}

Subjects

A total of 24 manual WCUs, 22 men and 2 women (mean \pm s.d.; age, $39.2 \pm 10.9$ years; height, $174 \pm 9 \mathrm{~cm}$; weight, $72.9 \pm 10.9 \mathrm{~kg}$; body mass index,

\footnotetext{
${ }^{1}$ Medical and Scientific Department, International Paralympic Committee, Bonn, Germany; ${ }^{2}$ Laboratory of Exercise Physiology, Department of Health and Human Performance, Faculty of Physical Activity and Sport Sciences, Technical University of Madrid, Madrid, Spain and ${ }^{3}$ Research Center on Physical Disability, ASPAYM Castilla y León Foundation, Valladolid, Spain

Correspondence: I Rossignoli, Medical and Scientific Department, International Paralympic Committee, Adenauerallee 212-214, 53113 Bonn, Germany.

E-mail: isabelrossignoli@gmail.com

Received 13 April 2014; revised 17 October 2014; accepted 24 October 2014; published online 25 November 2014
} 

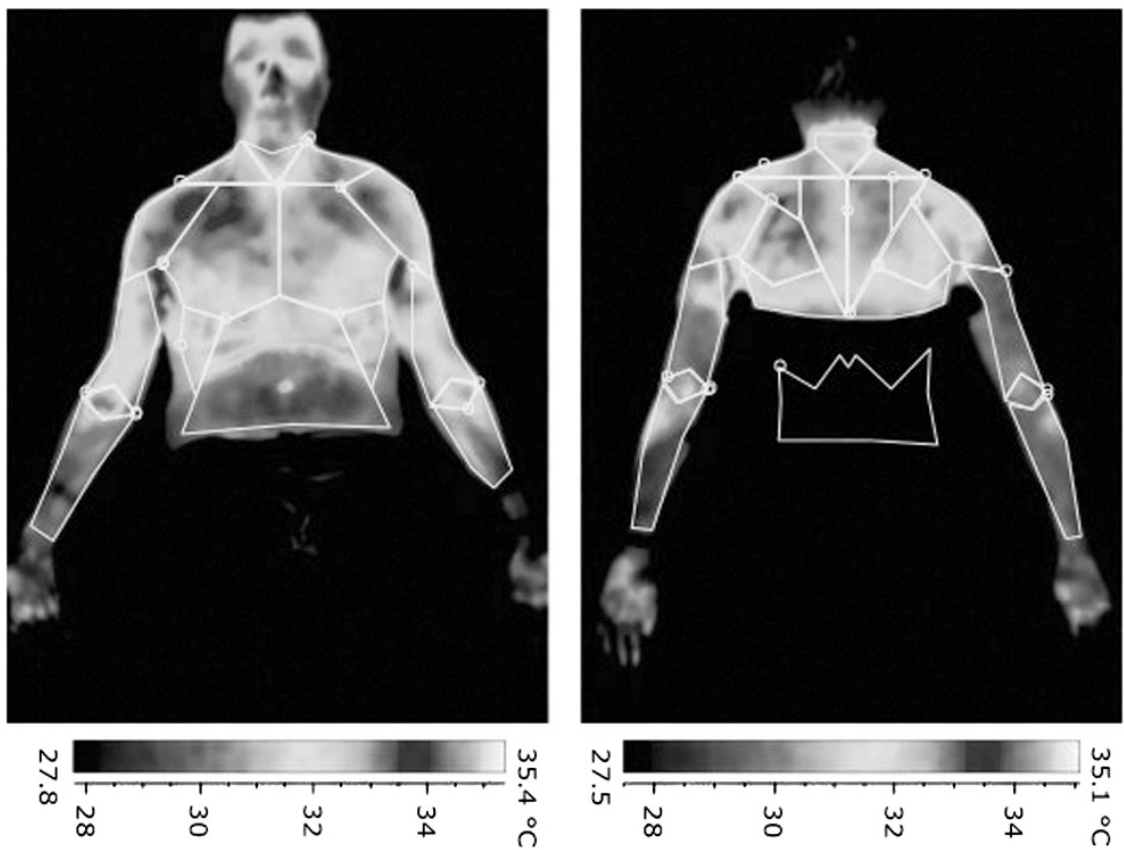

Figure 1 Distribution of the 36 regions of Interest in the anterior (left) and posterior (right) sides of the body.

$24.2 \pm 3.3 \mathrm{~kg} \mathrm{~m}^{-2}$ ), participated voluntarily in this study. In all, 19 were manual-wheelchair dependent and 5 were wheelchair independent (use of wheelchair only to practice sport), 14 were subjects with paraplegia, 3 with tetraplegia and 7 presented other disabilities (amputation, multiple sclerosis and poliomyelitis). Prior to the investigation, subjects were fully familiarized with testing procedures, were informed about the risks and benefits of the study, and signed an institutionally approved informed consent document. The protocol was approved by the Ethical Committee of the Technical University of Madrid and the Research Center of Physical Disability (CIDIF) of ASPAYM Castilla y León Foundation. Inclusion criteria were manual-wheelchair use for a period of at least 1 year and age $>18$ years.

\section{IRT analysis}

Infrared thermal imaging of the upper extremities and the trunk was recorded using a thermal camera (FLIR T335, FLIR Systems, Danderyd, Sweden), with a thermal sensitivity of $50 \mathrm{mK}$, a range of temperature from $-20^{\circ} \mathrm{C}$ to $+120^{\circ} \mathrm{C}$, a length spectrum range of $7.5-13 \mu \mathrm{m}$, a resolution of $320 \times 240$ pixels and an accuracy of $\pm 2 \%$. The camera was calibrated before each trial using an external temperature reference source (thermistor PT-100, Telemeter Electronic, Donauworth, Germany) and was turned on $20^{\prime}$ before to avoid calibration problems. The IRT images were analyzed with specific software (ThermaCAM Reporter, version 8, FLIR Systems). A white 'Roll-up' $125 \times 206 \mathrm{~cm}$ was used to create a homogeneous background behind body figures and to avoid the radiation emitted by other objects. A 'step' was placed for slightly raising the subject from the floor surface. The environmental conditions were monitored by a portable weather station (BAR-908-HG model, Oregon Scientific, Portland, OR, USA).

\section{Experimental procedure}

Two thermographic photographs corresponding to the anterior and posterior upper body were taken over 2 days with an interval of 1 day. All images were taken and analyzed by the same observer. Images were taken with the subject's own daily wheelchair in anatomical position. The camera was positioned perpendicular to the ground, and the distance between the subject and the infrared camera was fixed at 2-3 m, depending on the height of subjects, as the aim was to match the center of the image with the geometric center of the area to be evaluated. A transparent template helped the technician for the placement of the camera. Each image was manually divided into different body areas (Figure 1) on the basis of previous studies. ${ }^{7,10,11}$ Several body areas were eliminated from the analysis where: the lumbar area was hidden by the wheelchair, the abdomen and both flanks were not considered a relevant muscular area as many subjects with spinal cord injuries do not have muscle tone in that area, elbows and cubital fosses areas are not important for the propulsion of the wheelchair, the pectoral data of women were discarded owing to the influence of their bra.

The circadian-rhythm effect over Tsk ${ }^{12}$ was avoided by always testing each subject at the same hour of the day. During the first session, the subject responded verbally to a demographic and medical questionnaire. Before every thermographic examination subjects were instructed to refrain from the following for $24 \mathrm{~h}$ : applying creams or perfume, smoking, drinking alcohol or coffee, sunbathing, showering, receiving treatment, therapy, massage or undertaking exercise. All subjects postponed their weekly therapies after the thermographic tests, and they were asked not to push their wheelchairs before the test more than was strictly necessary. Prior to the assessment, subjects were asked to remain at rest, without clothes and jewelry on the upper body for at least $10 \mathrm{~min}$ before being photographed. This acclimatization period allowed the subjects' Tsk to stabilize. ${ }^{13}$ Every test was performed under equal conditions and in the same room, in which the temperature was fixed at $23^{\circ} \mathrm{C}$ and drafts were eliminated.

\section{Statement of ethics}

We certify that all applicable institutional and governmental regulations concerning the ethical use of human volunteers were followed during the course of this research.

\section{Statistical analysis}

After applying the Kolmogorov-Smirnov tests with the Lilliefors correction, asymmetry and kurtosis, \pm 1 and \pm 2 , respectively, ${ }^{14,15}$ the normality of the dependent variables was verified and parametric statistics were therefore applied.

ICC was used to analyze the reliability and coefficient of variation (CV; s.d. ${ }^{*} 100$ per mean) to analyze the dispersion data. ICC was characterized as follows: ${ }^{16}$ poor reproducibility, $0-0.39$; fair reproducibility, $0.40-0.59$; good reproducibility, 0.60-0.74; excellent reproducibility, 0.75-1.0. Descriptive statistics were used (mean \pm s.d.) to describe, organize and summarize the data for the number of assessments (day 1 and day 2). Side-to-side skin temperature differences $(\delta \mathrm{Tsk})$ were calculated for each area by subtracting the mean temperature of the maximum values of the right side from that of the left side. 
The minimum difference was calculated for all the body areas to study the relevancy of day-to-day Tsk. Student's $t$-test for related samples was used to ascertain whether there were any differences across the two measures. The statistical treatment of data was performed using SPSS 19.0 software for Windows (SPSS Inc., Chicago, IL, USA). The level of significance was set at $P \leqslant 05$ for all statistical analyses.

\section{RESULTS}

\section{Reliability of measurements}

A total of 1728 images for the trunk and upper extremities were recorded from 24 subjects. By comparing the assessment of day 1 and day 2 for the temperature profile, no significant differences were observed in any of the 36 zones, or in the mean or maximum values. The only differences were observed in the average temperature of the right posterior arm $(P=0.041)$, with a difference of $2.05^{\circ} \mathrm{C}$ between day 1 and day 2 .

The best maximum values of ICC were found in the left anterior forearm, right posterior forearm, left posterior arm and left anterior

Table 1 Descriptive maximum values of the temperature profile on day 1 , day 2 and between both the assessments of the analyzed area

\begin{tabular}{|c|c|c|c|c|c|}
\hline \multirow{2}{*}{$\begin{array}{l}\text { Region of } \\
\text { interest }\end{array}$} & \multirow{2}{*}{$\begin{array}{l}\text { Day } 1 \\
\text { X土s.d. }\end{array}$} & \multirow{2}{*}{$\begin{array}{l}\text { Day } 2 \\
\text { X土s.d. }\end{array}$} & \multicolumn{3}{|c|}{ Day 1 and 2} \\
\hline & & & $C V$ & $I C C$ & Scale \\
\hline RafM & $33.31 \pm 1.36$ & $33.38 \pm 1.16$ & 1.56 & 0.6 & Good \\
\hline LafM & $33.10 \pm 1.17$ & $33.00 \pm 1.13$ & 1.23 & 0.76 & Excellent \\
\hline RaaM & $33.96 \pm 0.75$ & $33.97 \pm 0.99$ & 1.36 & 0.52 & Fair \\
\hline LaaM & $33.50 \pm 0.72$ & $33.53 \pm 0.92$ & 1.14 & 0.58 & Fair \\
\hline ANM & $33.67 \pm 0.84$ & $33.79 \pm 0.77$ & 1.13 & 0.56 & Fair \\
\hline RasM & $34.15 \pm 0.65$ & $34.21 \pm 0.83$ & 1.17 & 0.52 & Fair \\
\hline LasM & $33.90 \pm 0.72$ & $33.85 \pm 1.19$ & 1.51 & 0.49 & Fair \\
\hline RpM & $34.29 \pm 0.62$ & $34.39 \pm 0.83$ & 1.05 & 0.59 & Fair \\
\hline LpM & $34.19 \pm 0.61$ & $34.24 \pm 1.00$ & 1.5 & 0.39 & Poor \\
\hline RatM & $34.12 \pm 0.71$ & $33.93 \pm 1.36$ & 1.5 & 0.53 & Fair \\
\hline LatM & $33.91 \pm 1.01$ & $33.98 \pm 1.17$ & 1.12 & 0.79 & Excellent \\
\hline RpfM & $32.94 \pm 1.53$ & $33.15 \pm 1.65$ & 1.54 & 0.77 & Excellent \\
\hline LpfM & $33.06 \pm 1.68$ & $33.20 \pm 1.47$ & 1.8 & 0.69 & Good \\
\hline RpaM & $32.63 \pm 0.83$ & $32.90 \pm 1.04$ & 1.62 & 0.46 & Fair \\
\hline LpaM & $32.55 \pm 1.28$ & $32.69 \pm 1.20$ & 1.44 & 0.77 & Excellent \\
\hline PNM & $33.54 \pm 0.76$ & $33.61 \pm 0.91$ & 1.31 & 0.53 & Fair \\
\hline RdoM & $33.01 \pm 0.99$ & $33.07 \pm 1.22$ & 1.57 & 0.59 & Fair \\
\hline LdoM & $33.01 \pm 0.69$ & $33.10 \pm 1.20$ & 1.51 & 0.54 & Fair \\
\hline RpsM & $32.81 \pm 1.09$ & $32.70 \pm 1.73$ & 2.04 & 0.6 & Good \\
\hline LpsM & $33.17 \pm 0.72$ & $33.24 \pm 1.03$ & 1.41 & 0.54 & Fair \\
\hline RpiM & $33.14 \pm 0.88$ & $33.35 \pm 1.22$ & 1.44 & 0.67 & Good \\
\hline LpiM & $33.27 \pm 0.86$ & $33.42 \pm 1.15$ & 1.47 & 0.6 & Good \\
\hline RpsuM & $33.27 \pm 0.75$ & $33.47 \pm 0.99$ & 1.27 & 0.65 & Good \\
\hline LpsuM & $33.45 \pm 0.82$ & $33.53 \pm 1.05$ & 1.37 & 0.59 & Fair \\
\hline CRptM & $33.85 \pm 0.77$ & $33.91 \pm 0.95$ & 1.47 & 0.49 & Fair \\
\hline CLptM & $33.72 \pm 0.89$ & $33.75 \pm 1.01$ & 1.49 & 0.52 & Fair \\
\hline RptM & $33.58 \pm 0.79$ & $33.62 \pm 1.01$ & 1.26 & 0.64 & Good \\
\hline LptM & $33.61 \pm 0.92$ & $33.61 \pm 1.03$ & 1.48 & 0.58 & Fair \\
\hline X & & & 1.42 & 0.59 & Fair \\
\hline
\end{tabular}

Abbreviations: aa, anterior arm; af, anterior forearm; AN, anterior area of the neck; as, anterior shoulder; at, anterior trapezius; CLpt, central-left posterior trapezius; CRpt, central-right posterior trapezius; CV, coefficient of variation; do, dorsal muscle area; ICC, coefficient of intraclass correlation; $\mathrm{p}$, pectoral; pa, posterior arm; pf, posterior forearm; pi, posterior infraspinatus muscle area; PN, posterior area of the neck or cervical area; ps, posterior shoulder; psu, posterior supraspinatus muscle area; pt, posterior trapezius.

NOTE: Values are mean \pm s.d. Scale refers to the scale of reproducibility of Cicchetti and Sparrow. ${ }^{16}$ Suffix ' $X$ ' corresponds to 'average' values. For the 'region of interest': prefix ' $L$ ' refers to the 'left side' and ' $R$ ' to the 'right side' of the variable. Suffix ' $M$ ' corresponds to the 'maximum' values. trapezius, with $0.76,0.77,0.77,0.79$, respectively (see Table 1 ). For the average values the highest ICC was the following: left posterior forearm, 0.80; right posterior arm, 0.88; right dorsal, 0.79; right pectoral, 0.95; left supraspinatus, 0.76 (see Table 2). Some zones presented a poor reproducibility: right anterior forearm, 0.22; left anterior forearm, 0.24; left anterior arm, 0.18; left posterior arm, 0.23; left anterior shoulder, 0.15; left pectoral, 0.39; LptX left posterior trapezius, 0.38. Hence, IRT in WCUs showed a poor to excellent reliability for the left posterior arm and right posterior arm (ICC $=$ 0.15 and 0.95 , respectively). For a graphical view of the day-to-day analysis of reliability see Figure 2.

The CV between day 1 and day 2 ranged between 1.05\% (RpM) and $6.18 \%$ (LptX). Table 1 shows the variability and the reliability of each variable (maximum values) and Table 2 presents the same descriptive values for variables that describe average values. The mean of the average values of all the areas studied was: $31.83 \pm 2.04$; CV, $2.80 \%$; ICC, 0.56 , with a $\delta$ Tsk between day 1 and 2 of $0.08^{\circ} \mathrm{C}$. The analysis of the main body areas (see Table 3 ) allows appreciating a lower Tsk in the limbs $(31.63 \pm 1.97)$ than in the trunk $(31.98 \pm 1.79)$, and a lower Tsk in the distal extremities (forearms colder than arms, arms colder than shoulders).

The maximum temperatures were taken as references to calculate the $\delta$ Tsk between symmetrical sides of the body. Table 4 shows the absolute values of mean \pm s.d.; the first and second columns are referred as $\delta$ Tsk between both sides of the body on day 1 and day 2; the third column is related to $\delta$ Tsk between the days. The $\delta$ Tsk did not show any consistent pattern related to the handedness of the subjects. The minimum difference of all the body areas was 0.576.

\section{DISCUSSION}

Before establishing the efficacy of IRT as a tool for assessing the evolution of pain, it is necessary to evaluate its reliability in order for it to be implemented on populations with diverse characteristics. The main contribution of this study is that its reliability is highly dependent on the area to be analyzed. IRT in WCUs had a variable ICC and CV, and it demonstrated a poor to excellent reliability (ICC: LasX $=0.15 ; \mathrm{RpX}=0.95$ ). This range of reliabilities must be taken into account when interpreting thermographic data.

To our knowledge, there is only one study investigating IRT and Tsk evaluation of WCUs. ${ }^{17}$ It found that the temperature in the palm, forearm, pectoral major and shoulder tended to increase during wheelchair propulsion compared with other parts of the trunk. In the posterior trunk the warmest areas corresponded to the trapezius and forearms. ${ }^{17}$ Our results are similar, as the trunk was warmer than the limbs, and the forearm and shoulder were warmer than the anterior trunk, but it is not possible to compare both investigations as our measurements were undertaken while the subjects were at rest.

There are no previous studies about the reliability of IRT as a means of assessing body temperature in WCUs, although some researchers have studied the IRT reliability of non-WCUs. Our study is focused on works that studied similar areas as ours (the upper body except the hands, wrists and lumbar areas). In a study by Littlejohn et al. ${ }^{18}$ thermal ICC values for the forearm ranged from 0.19 to 0.85 . Zaproudina et al. ${ }^{5}$ analyzed the whole body and found a mean ICC of 0.47. Most of the areas with low ICC that they analyzed are coincident with our study, whereupon it is necessary to pay attention to those relevant areas (trapezius, forearm and shoulder). Burnham et al. ${ }^{8}$ found a high reliability (ICC 0.97) in the hand, forearm, shoulder, thigh, shin and foot. Several studies, ${ }^{6,19-21}$ about paraspinal Tsk discovered a fair to excellent intra-examiner reproducibility (ICC 0.51-0.98). However, the surface of some analyzed areas differs slightly 
Table 2 Descriptive average values of the temperature profile on day 1 , day 2 and between both the assessments for each analyzed area

\begin{tabular}{|c|c|c|c|c|c|}
\hline \multirow{2}{*}{$\begin{array}{l}\text { Region of } \\
\text { interest }\end{array}$} & \multirow{2}{*}{$\begin{array}{l}\text { Day } 1 \\
\text { X士 s.d. }\end{array}$} & \multirow{2}{*}{$\begin{array}{l}\text { Day } 2 \\
\text { X士s.d. }\end{array}$} & \multicolumn{3}{|c|}{ Day 1 and 2} \\
\hline & & & $\mathrm{CV}$ & $I C C$ & Scale \\
\hline RafX & $31.26 \pm 2.54$ & $31.40 \pm 2.35$ & 3.56 & 0.24 & Poor \\
\hline LafX & $31.8 \pm 2.63$ & $31.19 \pm 2.45$ & 3.65 & 0.24 & Poor \\
\hline RaaX & $32.42 \pm 0.96$ & $32.19 \pm 1.25$ & 1.58 & 0.57 & Fair \\
\hline LaaX & $30.93 \pm 4.20$ & $32.19 \pm 1.15$ & 3.14 & 0.18 & Poor \\
\hline ANX & $32.44 \pm 1.21$ & $32.84 \pm 1.21$ & 1.63 & 0.67 & Good \\
\hline RasX & $33.09 \pm 0.87$ & $33.24 \pm 0.96$ & 1.45 & 0.56 & Fair \\
\hline LasX & $33.01 \pm 0.80$ & $32.10 \pm 3.94$ & 4.14 & 0.15 & Poor \\
\hline $\mathrm{RpX}$ & $31.14 \pm 2.91$ & $31.47 \pm 2.89$ & 1.61 & 0.95 & Excellent \\
\hline LpX & $31.99 \pm 1.57$ & $31.91 \pm 2.06$ & 2.39 & 0.62 & Good \\
\hline RatX & $32.54 \pm 3.00$ & $31.29 \pm 5.13$ & 4.66 & 0.6 & Good \\
\hline LatX & $30.65 \pm 5.51$ & $31.08 \pm 5.49$ & 4.84 & 0.69 & Good \\
\hline RpfX & $31.04 \pm 3.62$ & $31.55 \pm 2.72$ & 2.74 & 0.66 & Good \\
\hline LpfX & $30.95 \pm 3.91$ & $30.95 \pm 3.68$ & 2.48 & 0.8 & Excellent \\
\hline RpaX & $29.21 \pm 4.35$ & $31.25 \pm 1.35$ & 1.38 & 0.88 & Excellent \\
\hline LpaX & $29.89 \pm 3.12$ & $30.44 \pm 2.49$ & 5.17 & 0.23 & Poor \\
\hline PNX & $32.53 \pm 0.91$ & $32.73 \pm 1.05$ & 1.59 & 0.46 & Fair \\
\hline RdoX & $30.98 \pm 3.62$ & $30.28 \pm 4.13$ & 3.59 & 0.79 & Excellent \\
\hline LdoX & $31.27 \pm 1.82$ & $31.22 \pm 2.43$ & 3.16 & 0.5 & Fair \\
\hline RpsX & $31.13 \pm 2.92$ & $29.97 \pm 4.91$ & 5.14 & 0.58 & Fair \\
\hline LpsX & $32.12 \pm 0.83$ & $32.11 \pm 1.16$ & 1.66 & 0.51 & Fair \\
\hline RpiX & $32.28 \pm 1.10$ & $32.48 \pm 1.29$ & 2.01 & 0.51 & Fair \\
\hline LpiX & $32.48 \pm 0.91$ & $32.60 \pm 1.21$ & 1.65 & 0.55 & Fair \\
\hline RpsuX & $32.64 \pm 0.92$ & $32.89 \pm 1.13$ & 1.34 & 0.68 & Good \\
\hline LpsuX & $32.15 \pm 1.91$ & $31.95 \pm 2.48$ & 2.23 & 0.76 & Excellent \\
\hline CRptX & $31.90 \pm 1.95$ & $32.12 \pm 2.14$ & 2.06 & 0.73 & Good \\
\hline CLptX & $32.79 \pm 0.86$ & $32.92 \pm 1.16$ & 1.79 & 0.47 & Fair \\
\hline RptX & $32.92 \pm 0.83$ & $32.90 \pm 1.10$ & 1.47 & 0.63 & Good \\
\hline LptX & $31.57 \pm 3.96$ & $31.43 \pm 4.06$ & 6.18 & 0.38 & Poor \\
\hline$x$ & & & 2.80 & 0.56 & Fair \\
\hline \multicolumn{6}{|c|}{ 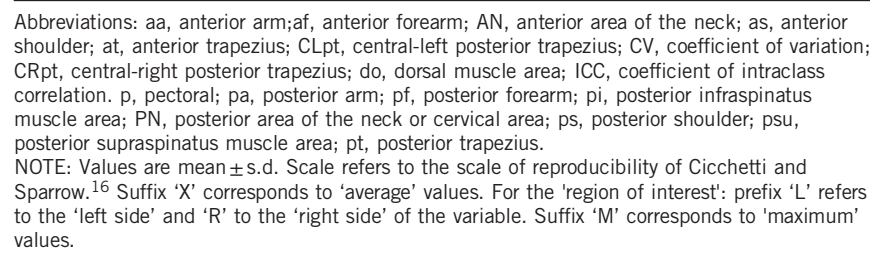 } \\
\hline
\end{tabular}

between studies, and, consequently, some areas with the same name in each study ${ }^{5,8}$ corresponded to different areas. Some studies only measured specific skin spots, because they used an infrared skin thermometer, ${ }^{8}$ a handheld thermographic scanner, ${ }^{19}$ or a handheld infrared paraspinal instrument ${ }^{21}$ instead of an infrared camera. Furthermore, the research by Plaugher et al. ${ }^{6}$ was undertaken with contact thermography, whereas IRT is noncontact thermography. Their research ${ }^{6}$ relied on the examiner's interpretation instead of a computerized reading of data, whereas other studies, including ours, manually measured the body areas and compared one graph with another.

Injury processes of some body areas are the main explanation for the fluctuations in the reliability, as the temperature varies depending on the type and moment of the injury (post-traumatic pain syndrome and sympathetic nerve involvement result in vasoconstriction and a colder area; acute and inflammatory injury increase metabolism, blood flow and dermal temperatures). ${ }^{22}$ The particular characteristics of

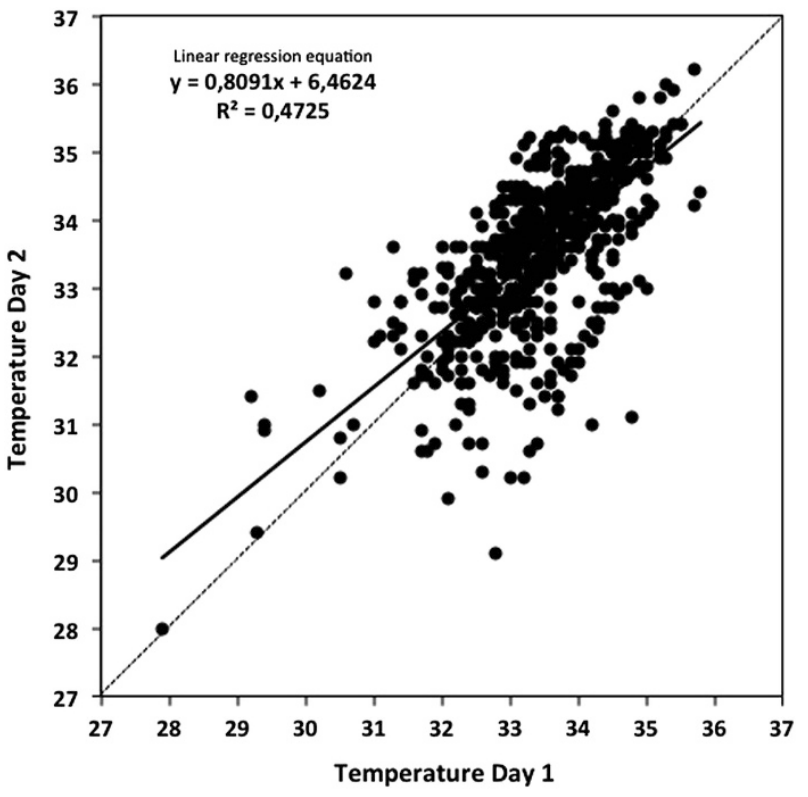

Figure 2 Day-to-day analysis of the reliability in all body areas measured.

Table 3 Interrater-reliability measures in terms of ICC and CV organized by general areas

\begin{tabular}{|c|c|c|c|c|c|}
\hline \multirow[t]{2}{*}{ Body area } & \multicolumn{3}{|c|}{ Average values } & \multicolumn{2}{|c|}{ Maximum values } \\
\hline & Mean \pm s.d. & $\mathrm{CV}$ & ICC & $\mathrm{CV}$ & ICC \\
\hline Arm & $31.32 \pm 2.09$ & 2.96 & 0.48 & 1.46 & 0.64 \\
\hline Shoulder & $32.10 \pm 1.86$ & 3.10 & 0.45 & 1.53 & 0.54 \\
\hline Trapezious & $31.80 \pm 3.25$ & 4.29 & 0.57 & 1.34 & 0.63 \\
\hline Pectoral & $31.63 \pm 2.26$ & 2.00 & 0.79 & 1.28 & 0.49 \\
\hline Back & $32.07 \pm 1.67$ & 2.23 & 0.62 & 1.45 & 0.58 \\
\hline Neck & $32.63 \pm 0.97$ & 1.61 & 0.56 & 1.22 & 0.54 \\
\hline
\end{tabular}

Abbreviations: $\mathrm{CV}$, coefficient of variation; ICC, coefficient of intraclass correlation.

people with disabilities may have also influenced the reliability, such as the thermoregulation problems presented by the subjects with $\mathrm{SCI}^{23}$ and sweating characteristics of multiple sclerosis subjects. ${ }^{24}$ People with SCI have a loss of autonomic nervous system control for vasomotor and sudomotor responses below the level of SCI, and a reduced thermoregulatory effector response for a given core temperature. ${ }^{25,26}$ As a consequence of their poikilothermic behavior, they have a reduced ability to tolerate thermal extremes, ${ }^{26}$ they have increased heat storage within the lower body ${ }^{25}$ and they tend to sweat less. $^{25}$ This imbalance in temperature regulation (most pronounced in individuals with tetraplegia rather than paraplegia) makes Tsk very changeable from one day to another, influencing the reliability of our data. Other factors that may locally change the blood flow and the Tsk are the variation of the peripheral circulation in the distal parts of the body ${ }^{5}$ obliterating artery disease, malignancies, varicose veins or inflammatory processes. ${ }^{27}$

The distribution of the upper body temperature in our study was similar to the literature. ${ }^{8,28}$ Central sites were warmer than peripheral sites, and this may be explained by blood distribution. ${ }^{28}$ Unlike Zhu and $\mathrm{Xin}^{28}$ the subjects in our study had a slightly higher ventral body temperature $(31.92 \pm 2.22)$ than dorsal body temperature $(31.77 \pm 1.92)$. Commonly the ventral area has greater skin thickness 
Table 4 The mean maximal values of regional skin temperature $\left({ }^{\circ} \mathrm{C}\right.$ ) and absolute values of $\delta \mathrm{T}$ (side-to-side temperature differences) with the standard deviations for the different skin areas of the study subjects

\begin{tabular}{|c|c|c|c|c|c|c|}
\hline \multirow[t]{2}{*}{ Body area } & \multirow{2}{*}{$\begin{array}{c}\delta T \text { Day } 1 \\
\text { mean } \pm \text { s.d. }\end{array}$} & \multirow{2}{*}{$\begin{array}{c}\delta T \text { Day } 2 \\
\text { mean } \pm \text { s.d. }\end{array}$} & \multicolumn{2}{|c|}{$\delta T$ Between days } & \multirow[t]{2}{*}{$r$} & \multirow[t]{2}{*}{ P-value } \\
\hline & & & mean \pm s.d. & P-value & & \\
\hline A forearm & $0.12 \pm 0.66$ & $0.39 \pm 0.65$ & $0.27 \pm 0.75$ & 0.117 & 0.352 & 0.118 \\
\hline A arm & $0.42 \pm 0.41$ & $0.35 \pm 0.49$ & $0.07 \pm 0.43$ & 0.479 & 0.540 & $0.008^{*}$ \\
\hline A shoulder & $0.24 \pm 0.30$ & $0.16 \pm 0.29$ & $0.08 \pm 0.20$ & 0.081 & 0.778 & $0.001^{\dagger}$ \\
\hline Pectoral & $0.13 \pm 0.29$ & $0.07 \pm 0.38$ & $0.06 \pm 0.27$ & 0.287 & 0.703 & $0.001^{\dagger}$ \\
\hline A trapezius & $0.18 \pm 0.72$ & $0.01 \pm 0.28$ & $0.17 \pm 0.74$ & 0.283 & 0.146 & 0.517 \\
\hline$P$ forearm & $0.05 \pm 0.61$ & $0.05 \pm 0.59$ & $0.01 \pm 0.53$ & 0.969 & 0.609 & $0.003^{*}$ \\
\hline $\mathrm{P}$ arm & $0.17 \pm 0.78$ & $0.22 \pm 0.74$ & $0.05 \pm 0.72$ & 0.752 & 0.553 & $0.006^{*}$ \\
\hline Dorsal & $0.00 \pm 0.48$ & $0.03 \pm 0.36$ & $0.03 \pm 0.42$ & 0.737 & 0.536 & $0.007 *$ \\
\hline P shoulder & $0.40 \pm 1.06$ & $0.31 \pm 1.07$ & $0.09 \pm 0.35$ & 0.237 & 0.946 & $0.001^{\dagger}$ \\
\hline Infraspinatus & $0.13 \pm 0.47$ & $0.07 \pm 0.50$ & $0.06 \pm 0.29$ & 0.335 & 0.821 & $0.001^{\dagger}$ \\
\hline Supraspinatus & $0.18 \pm 0.48$ & $0.06 \pm 0.43$ & $0.12 \pm 0.38$ & 0.137 & 0.648 & $0.001^{*}$ \\
\hline P central Trapezius & $0.12 \pm 0.33$ & $0.12 \pm 0.30$ & $0.02 \pm 0.25$ & 0.745 & 0.679 & $0.001^{\dagger}$ \\
\hline P trapezius & $0.08 \pm 0.29$ & $0.07 \pm 0.33$ & $0.15 \pm 0.38$ & 0.078 & 0.269 & 0.226 \\
\hline Total X & $0.17 \pm 0.53$ & $0.15 \pm 0.49$ & $0.09 \pm 0.44$ & & 0.583 & \\
\hline
\end{tabular}

Abbreviations: A, anterior; $\mathrm{P}$, posterior; $r$, Pearson correlation coefficient; $\mathrm{X}$, average.

Abbreviations: A, anterior; P, post
NOTE: Values are mean \pm s.d.

Diagnostic accuracy of IRT using thermographic asymmetry.

${ }^{*} P<0.05$.

${ }^{\dagger} P<0.001$.

than the dorsal area, and subcutaneous fat in the area is known to influence the thermal readings. ${ }^{18}$

The mean ICC for average values (Table 1) is 0.56 (fair). When areas with lower ICC values are removed (the forearm, arm, shoulder and trapezius) the mean ICC augments to 0.64 (good). For the maximum values (Table 2) the mean ICC is 0.59 (fair) and without the most troubled area, the pectoral, the mean ICC becomes 0.60 (good). These areas that are sensitive to error are involved in wheelchair propulsion; we suggest the participant is carried to the room to avoid doing any exercise before the testing. The average values have a greater number of areas with poor ICC than the maximum values. When regions of interest are manually drawn, certain limits can go out of the boundary and encompass part of the background picture, and hence the average temperature of this area would be lower. The maximum values are not affected by extreme values, and thus they are more congruent; therefore, they were chosen as references to calculate the $\delta$ Tsk between symmetrical sides of the body.

Burnham et al. ${ }^{8}$ affirmed that side-to-side temperature comparisons could be used as a measure of an instrument's reliability. Most of the articles found $\delta$ Tsk or thermal asymmetry of around $0.5^{\circ} \mathrm{C}, 8,290.4^{\circ} \mathrm{C}^{5}$ and $0.3^{\circ} \mathrm{C} .11,30$ Our data showed a $\delta$ Tsk for maximum values of: $0.17 \pm 0.53^{\circ} \mathrm{C}$ and $0.15 \pm 0.49^{\circ} \mathrm{C}$ on days 1 and 2 , respectively, with a $\delta$ Tsk between both days of $0.09 \pm 0.44^{\circ} \mathrm{C}$. s.d. values over $2.5-3$ indicate a Tsk abnormality in certain part of their bodies. ${ }^{29}$ There were no significant differences between day 1 and day 2. The correlation was good for the anterior and posterior arm, posterior forearm, dorsal, supraspinatus and central trapezius, and was excellent for the anterior and posterior shoulder, pectoral and infraspinatus. The average correlation was moderate $(r=0.583)$. Low correlation was found in the anterior forearm, anterior and posterior trapezius. Previous literature proposes different reasons that could cause these variations, such as Owens et al., ${ }^{19}$ who suggested that changes could be explained by physiological variations rather than equipment error.

\section{Study limitations}

A manual system was used to draw the limits of each area. Consequently, the demarcation lines of the examined area could vary slightly from one picture to another. The data may be affected by temporary injuries in the absence of a prior physical examination. The time interval between both measurements was reduced to one day to minimize the thermal evolution of potential lesions, given that the initial warm areas may quickly return to normal temperatures. ${ }^{7}$ However, this time interval is still wide and gives the highest error of reliability possible, because it measures many factors that may affect the temperature. We propose taking pictures on the same day to isolate the reliability from other factors.

\section{CONCLUSIONS}

This study showed that the reliability varies by anatomic regions of the body. The infrared technique evaluated in this study is fast, easy to use and it seems to be adequately reliable to monitor Tsk in the population studied. Particular considerations of each subject that affect Tsk values should be weighed, such as physiological variability of the blood flow, thermoregulation problems, hormonal changes, medication intake, scars or body fat. We recommend assessing the Tsk of WCUs in a standard wheelchair to avoid different backrest heights. Moreover, other technical factors (for instance, equipment accuracy, measurement environment or processing and interpretation of data) should be considered.

Further investigation is necessary to reproduce this study with different time intervals and to examine the validity and reliability of these temperature techniques in other indoor settings and exercise modes. Longitudinal prospective studies of IRT reliability on specific health conditions with larger and more homogeneous samples could help to explore the clinical application of the IRT on those pathologies. Future research should aim to associate the Tsk of the muscles involved in wheelchair propulsion with the pain in those areas, to prevent situations of injury risk. 


\section{DATA ARCHIVING}

There were no data to deposit.

\section{CONFLICT OF INTEREST}

The authors declare no conflict of interest.

\section{ACKNOWLEDGEMENTS}

We acknowledge Manuel Sillero-Quintana, $\mathrm{PhD}$, Director of the pemaSiP (INEF-UPM) research group, for providing us the thermographic camera. Special thanks to Pedro J Marín, Juan Martín and Héctor Menéndez for their technical help during the data collection. We thank the members of ASPAYM Castilla y León, the basketball players of MUPLI from Palencia and Grupo Norte Foundation from Valladolid, who voluntarily participated in this study.

1 Thurston NM, Kent B, Jewell MJ, Blood H. Thermographic evaluation of the painful shoulder in the hemiplegic patient. Phys Ther 1986; 66: 1376-1381.

2 Jones BF, Plassmann P. Digital infrared thermal imaging of human skin. IEEE Eng Med Biol Mag 2002; 21: 41-48.

3 Jiang LJ, Ng EY, Yeo AC, Wu S, Pan F, Yau WY et al. A perspective on medical infrared imaging. J Med Eng Technol 2005; 29: 257-267.

4 Sillero QM, Gómez CPM, Noya SJ, Núñez FJ, Fernández RI (eds). Comparation of Infrared Thermography with Tensiomyography as Injury Prevention Method in Professional Soccer Players. BASES. Taylor \& Francis: Leeds, 2009.

5 Zaproudina N, Varmavuo V, Airaksinen O, Narhi M. Reproducibility of infrared thermography measurements in healthy individuals. Physiol Meas 2008; 29: 515-524.

6 Plaugher G, Lopes MA, Melch PE, Cremata EE. The inter- and intraexaminer reliability of a paraspinal skin temperature differential instrument. J Manipulative Physiol Ther 1991; 14: 361-367.

7 Uematsu S. Thermographic imaging of cutaneous sensory segment in patients with peripheral nerve injury. Skin-temperature stability between sides of the body. J Neurosurg 1985; 62: 716-720.

8 Burnham RS, McKinley RS, Vincent DD. Three types of skin-surface thermometers: a comparison of reliability, validity, and responsiveness. Am J Phys Med Rehabil 2006; 85: 553-558.

9 Serrano I. Effect of Endurance, Speed and Strength Training on Skin Temperature Measured by Infrared Thermography. Technical University of Madrid, 2012.

10 Ammer K. The Glamorgan Protocol for recording and evaluation of thermal images of the human body. Thermology International 2008; 18: 125-129.

11 Uematsu S, Edwin DH, Jankel WR, Kozikowski J, Trattner M. Quantification of therma asymmetry. Part 1: Normal values and reproducibility. J Neurosurg 1988; 69: $552-555$
12 Miyakoshi N, Itoi E, Sato K, Suzuki K, Matsuura H. Skin temperature of the shoulder: circadian rhythms in normal and pathologic shoulders. J Shoulder Elbow Surg 1998; 7 625-628.

13 Ring E, Ammer K. The Technique of Infra red Imaging in Medicine. Thermol Int 2000; 10: 7-14.

14 Carter J, Jeukendrup AE. Validity and reliability of three commercially available breathby-breath respiratory systems. Eur J Appl Physiol 2002; 86: 435-441.

15 Martínez-González MA, De Irala-Estévez J, Faulin-Fajardo FJ. Bioestadística amigable, 1 st ed. Díaz de Santos, 2001.

16 Cicchetti DV, Sparrow SA. Developing criteria for establishing interrater reliability of specific items: applications to assessment of adaptive behavior. Am J Ment Defic 1981; 86: 127-137.

17 Shin-ichi W, Takashi M, Katsumi T, Takako S, Keijiro Y. Measurement of skin temperature during wheelchair exercise [in Japanese]. The Japan Society of Mechanical Engineers Symposium on Welfare Engineering 2005; 2005: 245-246.

18 Littlejohn RAN. Thermographic Assessment of the Forearm During Data Entry Tasks: A Reliability Study. Virginia Tech, 2008.

19 Owens EF Jr., Hart JF, Donofrio JJ, Haralambous J, Mierzejewski E. Paraspinal skin temperature patterns: an interexaminer and intraexaminer reliability study. J Manipulative Physiol Ther 2004; 27: 155-159.

20 McCoy M, Campbell I, Stone P, Fedorchuk C, Wijayawardana S, Easley K. Intra-examiner and inter-examiner reproducibility of paraspinal thermography. PLoS ONE 2011 February 11, 2011 6: e16535. Available from http://www.ncbi.nlm.nih.gov/ pmc/articles/PMC3037936/pdf/pone.0016535.pdf.

21 DeBoer KF, Harmon RO, Chambers R, Swank L. Inter- and intra-examiner reliability study of paraspinal infrared temperature measurements in normal students. Res Forum 1985; 2: 4-12.

22 Sands WA, McNeal JR, Stone MH. Thermal imaging and gymnastics injuries: a means of screening and injury identification. Sci Gymnastics J 2011; 3: 5-12.

23 Price MJ, Campbell IG. Effects of spinal cord lesion level upon thermoregulation during exercise in the heat. Med Sci Sports Exerc 2003; 35: 1100-1107.

24 Ueno M, Tokunaga Y, Terachi S, Gondo K, Hara T. Asymmetric sweating in a child with multiple sclerosis. Pediatric neurology 2000; 23: 74-76.

25 Price MJ. Thermoregulation during exercise in individuals with spinal cord injuries. Sports Med 2006; 36: 863-879.

26 Sawka MN, Latzka WA, Pandolf KB. Temperature regulation during upper body exercise: able-bodied and spinal cord injured. Med Sci Sports Exerc. 1989; 21 S132-S140.

27 Wasner G, Schattschneider J, Baron R. Skin temperature side differences-a diagnostic tool for CRPS? Pain 2002; 98: 19-26.

28 Zhu WP, Xin XR. Study on the distribution pa ttern of skin temperature in normal Chinese and detection of the depth of early burn wound by infrared thermography. Ann NY Acad Sci 1999; 888: 300-313.

$29 \mathrm{Niu} \mathrm{HH}$, Lui PW, Hu JS, Ting CK, Yin YC, Lo YL et al. Thermal symmetry of skin temperature: normative data of normal subjects in Taiwan. Zhonghua Yi Xue Za Zhi (Taipei) 2001; 64: 459-468.

30 Hildebrandt C, Raschner C, Ammer K. An overview of recent application of medical infrared thermography in sports medicine in Austria. Sensors 2010; 10: 4700-4715. 